\title{
Susceptibilidad Antibiótica de Cepas de Escherichia coli en Crías de Alpaca con y sin Diarrea
}

\author{
Antibiotic Susceptibility of Escherichia coli Strains in Neonatal Alpacas \\ WITH AND WITHOUT DIARRHOEA \\ Manuel Barrios-Arpi ${ }^{1}$, Siever Morales C. ${ }^{2,4}$, Eglinton Villacaqui-Ayllon ${ }^{3}$
}

\section{Resumen}

El estudio tuvo como objetivo determinar la susceptibilidad antibiótica de aislados de Escherichia coli en crías de alpaca con y sin diarrea de las comunidades campesinas de la localidad de Lachocc, departamento de Huancavelica, Perú. Se analizaron hisopados rectales de 95 animales con diarrea y de 60 aparentemente sanos de 1 a 2 meses de edad. El 48.4\% (46/95) y el 60\% (36/60) de las muestras de animales con y sin diarrea fueron positivas al cultivo de E. coli. Estos aislados fueron sensibles a la mayoría de los antibacterianos, especialmente trimetoprim (98\%), gentamicina (95\%) y fosfomicina ( $88 \%$ ). La excepción fue la nitrofurantoína, cuyas cepas mostraron una baja susceptibilidad $(8.3 \%)$.

Palabras clave: E. coli, antibiótico, susceptibilidad, alpaca

\section{Abstract}

The present study aimed to determine the antimicrobial susceptibility of Escherichia coli isolates in neonatal alpacas with and without diarrhea in peasant communities in Lachocc, Huancavelica, Peru. Rectal swabs from alpacas with diarrhoea $(n=95)$ and from apparently normal alpacas $(n=60)$ of 1 to 2 months of age were analysed. The results showed that $48.4 \%$ (46/95) and 60\% (36/60) of samples from animals with and without diarrhea were positive to $E$. coli. The isolates were sensitive to most of the antibiotics, especially trimethoprim (98\%), gentamicin (95\%) and fosfomycin (88\%). The exception was nitrofurantoin, whose strains showed low susceptibility $(8.3 \%)$.

Key words: $E$. coli, antibiotic, susceptibility, alpaca

${ }^{1}$ Laboratorio de Patología Clínica y Biología Molecular, ${ }^{2}$ Laboratorio de Microbiología y Parasitología Veterinaria, Facultad de Medicina Veterinaria, Universidad Nacional Mayor de San Marcos, Lima, Perú

${ }^{3}$ Carrera de Medicina Veterinaria y Zootecnia, Universidad Científica del Sur, Lima, Perú

${ }^{4}$ E-mail: sieverm@hotmail.com

Recibido: 25 de agosto de 2015

Aceptado para publicación: 15 de enero de 2016 


\section{INTRODUCCIÓN}

La diseminación de resistencia antimicrobiana en bacterias ha surgido en los últimos años como un serio problema, resultando en la creación de programas de vigilancia, tanto en medicina humana como en veterinaria (Cohen, 2000; WHO, 2002; Lanz et al., 2003). El uso indiscriminado de los antimicrobianos para el tratamiento y prevención de enfermedades, así como en la promoción del crecimiento en la crianza de animales de granja permite el desarrollo de bacterias resistentes a los antimicrobianos, resultando en una amenaza para la salud pública (Lanz et al., 2003).

La colibacilosis es una enfermedad de frecuente ocurrencia en crías de alpaca y es causante de elevadas morbilidades y mortalidades durante las primeras semanas de vida (Ameghino, 1990). La enfermedad ocasiona desde cuadros leves como una infección entérica hasta cuadros de septicemia que llevan a la muerte temprana del animal (Ameghino y DeMartini, 1991). Uno de los principales agentes infecciosos causales de estas patologías, sobre todo en neonatos, son cepas patógenas de Escherichia coli, que a diferencia de cepas comensales no patógenas, contienen elementos génicos adicionales responsables de su patogenicidad (Dobrint et al., 2003). E. coli, bacteria anaerobia facultativa de la flora intestinal normal de animales y humanos, es causante de la colibacilosis o diarrea blanca en camélidos sudamericanos (CSA), y que en condiciones naturales, puede estar asociada con otros agentes patógenos como los rotavirus (Ameghino, 1990; Morales et al., 2007).

E. coli es sensible a muchos antibióticos; sin embargo, algunos de ellos tienen un mayor efecto sobre ciertos patotipos de la bacteria. Dependiendo del tipo de infección, los antimicrobianos sulfatrimetoprim, fluoroquinolonas y cefalosporinas de tercera generación se recomiendan generalmente para el tratamiento de infecciones causadas por E. coli no STEC (E. coli productora de toxina shiga) (Thielman y Guerrant, 1999). El antibiograma por método de difusión en disco es rápido y fácil de realizar, y proporciona una respuesta rápida para la terapia antimicrobiana (Amezcua et al., 2002; Teale et al., 2002; Hendriksen et al., 2008).

Los mecanismos de resistencia antibiótica en $E$. coli hacia diferentes antibacterianos son múltiples y continuamente descritos. Estos mecanismos se adquieren mediante mutaciones puntuales a nivel cromosómico o transferencia horizontal de material genético entre especies, facilitada por elementos móviles, tales como los integrones. Esta transferencia permite que los mecanismos se trasladen entre diferentes enteropatógenos y que se diseminen rápidamente a nivel mundial (Mosquito et al., 2011). Asimismo, Iroha et al. (2012) demostraron la existencia de cepas multirresistentes de $E$. coli, tanto en animales como en humanos Sin embargo, existe una relativa escasez de información respecto a la resistencia a los antimicrobianos de la E. coli en animales (Iroha et al., 2012). En uno de los pocos estudios en CSA se reporta que el 80 y $25 \%$ de cepas de $E$. coli aisladas de crías de alpacas con cuadros diarreicos y casos fatales demostraron resistencia a la neomicina y oxitetraciclina, respectivamente (Luna, 2012).

El objetivo del presente estudio fue determinar la resistencia antibiótica de aislados de E. coli en crías de alpaca aparentemente sanas y en otras con cuadros entéricos, abriendo la posibilidad de diseminación poblacional bacteriana entre animales ante un patrón similar de resistencia y susceptibilidad antimicrobiana entre animales enfermos y sanos.

\section{Materiales Y Métodos}

\section{Lugar de Ejecución}

El presente estudio se realizó entre enero y marzo de 2013 en el Centro de Investigación y Desarrollo de Camélidos Sudameri- 
canos Lachocc de la Universidad Nacional de Huancavelica (CIDCS Lachocc-UNH), ubicado en la localidad de Lachocc, a $32 \mathrm{~km}$ suroeste de la ciudad de Huancavelica. Es una zona andina subtropical, con altitudes que varían entre de 4225 a 4850 msnm.

\section{Recolección de Muestras}

Se trabajó con animales pertenecientes a las comunidades campesinas de la Universidad de Huancavelica. El número de muestras fue obtenido basado en el teorema del límite central y en base a ellos se recolectaron muestras fecales mediante hisopados del recto de 155 crías de alpaca Huacaya de 1 a 2 meses de edad. De estos, 95 estaban con diarrea y 60 estaban clínicamente sanos.

Los hisopados fueron preservados en medios de transporte Stuart y trasladados al laboratorio de microbiología de la Facultad de Medicina Veterinaria, Universidad Nacional Mayor de San Marcos, en Lima.

\section{Procesamiento y Análisis de Muestras}

Las muestras fueron cultivadas en caldos de enriquecimiento y agar Mc Conkey durante 18 a 24 horas a $37^{\circ} \mathrm{C}$. Las colonias fermentadoras de lactosa (LFC) de cada muestra con la apariencia típica de $E$. coli y colonias rodeadas por zonas claras de hemólisis fueron coloreadas con la tinción Gram; asimismo, se les hizo la identificación bioquímica a través de las pruebas de fermentación de lactosa, producción de glucosa, indol, urea, sulfuro-indol-motilidad (SIM) e $\mathrm{H}_{2} \mathrm{~S}$. Fueron incluidas, además, las pruebas bioquímicas de rojo metilo, VoguesProskauer, malonato, fenilalanina, gluconato y sorbitol (INS, 2005).

Las pruebas de sensibilidad se realizaron por el método de difusión en disco de Kirby-Bauer (Bauer et al., 1966). Las placas fueron incubadas por $24 \mathrm{~h}$ a $37{ }^{\circ} \mathrm{C}$ en aerobiosis o anaerobiosis, según la cepa identificada, y se midieron los halos de inhibición de desarrollo, interpretándolos de acuerdo a las tablas del Comité Nacional para Estándares de Laboratorio Clínico (NCCLS, 2003). La susceptibilidad de los microorganismos a los agentes antimicro-bianos se calificaron como sensible, intermedia o resistente.

Los antibióticos (discos de sensibilidad) utilizados se encuentran agrupados dentro de las familias de beta-lactámicos (fosfomicina), fluoroquinolonas (enrofloxacina, ciprofloxacina), aminoglucósidos (amikacina, gentamicina), tetraciclinas (oxitetraciclina), cefalosporinas de tercera generación (ceftriazona, ceftadizima) y antibióticos asociados (trimetoprim-sulfametoxazol, nitrofurantoína). Estos fueron elegidos por su eficacia, disponibilidad en el mercado y toxicidad. Las placas se incubaron por $24 \mathrm{~h}$ a $37{ }^{\circ} \mathrm{C}$.

\section{Análisis Estadístico}

En el presente estudio se siguió el criterio para muestras por conglomerados, debido a que en el sistema extensivo de producción de CSA los animales usualmente son agrupados en corrales y pastorean en pequeñas a medianas «puntas» distribuidas en una localidad. Se utilizó la prueba de Chi-cuadrado para determinar la asociación entre la susceptibilidad antimicrobiana y la condición sanitaria (sano o enfermo).

\section{Resultados}

Los resultados de laboratorio indicaron que el 48.4\% (46/95) de las muestras de hisopados rectales de crías de alpaca con diarrea y el $60 \%(36 / 60)$ de aquellas sin diarrea fueron positivas al cultivo de E. coli.

Los aislados de $E$. coli provenientes de animales enfermos y sanos fueron sensibles a la mayoría de los antibacterianos, con frecuencias de sensibilidad entre 59 a $98 \%$, a excepción de la nitrofurantoína, cuyas cepas mostraron una susceptibilidad de $8.5 \%$. Los 
Cuadro 1. Susceptibilidad antibiótica de aislados de Escherichia coli en crías de alpaca con y sin diarrea $(n=82)$ de comunidades campesinas de Huancavelica, Perú

\begin{tabular}{lccc}
\hline Antibiótico & $\begin{array}{c}\text { Sensible } \\
(\%)\end{array}$ & $\begin{array}{c}\text { Intermedio } \\
(\%)\end{array}$ & $\begin{array}{c}\text { Resistente } \\
(\%)\end{array}$ \\
\hline Trimetoprim- & 98.0 & - & 2.0 \\
sulfametoxazol & 95.0 & 2.5 & 2.5 \\
Gentamicina & 88.0 & 6.0 & 6.0 \\
Fosfomicina & 78.0 & 11.0 & 11 \\
Ceftadizina & 74.0 & 22.0 & 4.0 \\
Ciprofloxacino & 72.0 & 26.0 & 2.0 \\
Ceftriaxona & 63.0 & 28.0 & 9.0 \\
Oxitetraciclina & 59.0 & 29.0 & 12.0 \\
Amikacina & 39.0 & 50.0 & 11.0 \\
Enrofloxacina & 8.5 & 7.3 & 84.0 \\
Nitrofurantoína & & & \\
\hline
\end{tabular}

Cuadro 2. Susceptibilidad antibiótica de aislados de Escherichia coli en crías de alpaca según la ocurrencia de diarrea (enfermas, $n=46$; sanas, $n=36$ ) de comunidades campesinas de Huancavelica, Perú

\begin{tabular}{|c|c|c|c|c|c|c|}
\hline \multirow[t]{2}{*}{ Antibiótico } & \multicolumn{2}{|c|}{$\begin{array}{c}\text { Sensible } \\
(\%)\end{array}$} & \multicolumn{2}{|c|}{$\begin{array}{c}\text { Intermedio } \\
(\%)\end{array}$} & \multicolumn{2}{|c|}{$\begin{array}{l}\text { Resistente } \\
(\%)\end{array}$} \\
\hline & Enfermo & Sano & Enfermo & Sano & Enfermo & Sano \\
\hline $\begin{array}{l}\text { Trimetoprim- } \\
\text { sulfametoxazol }\end{array}$ & 97.8 & 97.2 & 0 & 0 & 2.2 & 2.0 \\
\hline Gentamicina & 93.5 & 97.2 & 4.3 & 0 & 2.2 & 2.8 \\
\hline Fosfomicina & 87.0 & 88.9 & 4.3 & 8.3 & 8.7 & 2.8 \\
\hline Ceftadizina & 76.0 & 80.6 & 13.0 & 8.3 & 11.0 & 11.1 \\
\hline Ceftriaxona & 71.7 & 72.2 & 28.3 & 22.2 & 0 & 5.6 \\
\hline Ciprofloxacino & $84.8^{\mathrm{a}}$ & $61.1^{\mathrm{b}}$ & 15.2 & 30.6 & 0 & 8.3 \\
\hline Amikacina & 65.2 & 63.9 & 19.6 & 28.8 & 15.2 & 8.3 \\
\hline Oxitetraciclina & 63.0 & 50.0 & 28.3 & 41.7 & 8.7 & 8.3 \\
\hline Enrofloxacina & 43.5 & 33.3 & 52.2 & 47.2 & 4.3 & 19.5 \\
\hline Nitrofurantoína & 10.9 & 5.6 & 10.9 & 2.8 & 78.2 & 91.6 \\
\hline
\end{tabular}


antibióticos con mayor sensibilidad (>80\%) fueron trimetoprim-sulfametoxazol (98\%), gentamicina $(95 \%)$ y fosfomicina $(88 \%)$ (Cuadro 1). El análisis estadístico reveló que solo el ciprofloxacino estuvo asociado a la condición sanitaria ( $\mathrm{p}<0.05$; Cuadro 2).

\section{Discusión}

La resistencia antibiótica observada en el presente estudio fue de baja frecuencia. La mayoría de los aislados de E. coli, tanto de animales sanos como enfermos, mostraron susceptibilidad a los antibióticos utilizados a excepción de la nitrofurantoína. El nivel de resistencia a este antibiótico coincide con otros estudios donde se utilizaron mayores espectros de antibióticos contra E. coli (Sayah et al., 2005; Zahraei y Farashi, 2006). Asimismo, se reporta alta sensibilidad a este antibiótico en pacientes humanos con infecciones del tracto urinario, mas no así en animales de producción (Gales et al., 2002; Fadda et al., 2005).

La alta resistencia a la nitrofurantoína podría explicarse por el reciente uso indiscriminado de esta droga en el campo y por la aplicación de dosis inapropiadas. Esto es sugerente por el patrón de susceptibilidad similar encontrado en ambos grupos, lo cual estaría indicando que tanto la resistencia y susceptibilidad a los antibióticos utilizados en el presente estudio circulen por conjugación genética (De Francesco et al., 2004).

La susceptibilidad antibiótica fue evidenciada de forma similar en animales con y sin diarrea, a excepción del ciprofloxacino. Altos niveles de sensibilidad fueron observados para trimetoprim (98\%), gentamicina (95\%), fosfomicina $(88 \%)$, ceftadizima $(78 \%)$, ciprofloxacino $(74 \%)$, ceftriazona $(72 \%)$, oxitetraciclina $(63 \%)$ y amikacina $(59 \%)$, coincidiendo con otros estudios de susceptibilidad antibiótica de cepas de E. coli patogénicas y no patogénicas (Luna et al., 2012).
El ciprofloxacino es un antibiótico de amplio espectro perteneciente a la familia de las fluoroquinolonas, y fue el único de los antibióticos que demostró diferencias estadísticas en el comportamiento contra $E$. coli entre animales sanos y enfermos. Estudios previos han reportado una alta susceptibilidad al ciprofloxacino en rumiantes (Gupta et al., 2011), así como una alta efectividad bactericida in vitro (Chalkley y Koornhof, 1985), por lo que sería uno de los antibióticos recomendados para el tratamiento de diarreas por E. coli en rumiantes incluyendo los CSA, conjuntamente con trimetroprim-sulfametoxazol, gentamicina, fosfomicina y ceftazidina que obtuvieron mayor perfil de sensibilidad en el estudio.

\section{ConClusiones}

- Las cepas de Escherichia coli en animales con y sin diarrea evidencian patrones de sensibilidad y resistencia similares, sugiriendo la circulación constante de las cepas entre poblaciones de animales sanos y enfermos.

- La mayoría de cepas aisladas, tanto de alpacas enfermas como sanas, mostraron una alta sensibilidad a los antibióticos en estudio, con excepción de la nitrofuratoína.

- Se encontró asociación significativa entre ciprofloxacino y la condición sanitaria.

\section{Literatura Citada}

1. Ameghino E, De Martini J. 1991. Mortalidad de crías de alpacas. Bol Div IVITA Lima. p 71-80.

2. Ameghino E. 1990. Avances sobre investigación en salud animal: camélidos sudamericanos. Bol Div IVITAUNMSM 23: 1-66.

3. Amezcua R, Friendship RM, Dewey CE, Gyles C, Fairbrother JM. 2002. Presentation of postweaning 
Escherichia coli diarrhea in Southern Ontario, prevalence of haemolytic E. coli serogroups involved, and their antimicrobial resistance patterns. Can J Vet Res 66: 73-78.

4. Bauer A, Kirby W, Sherris J, Turck M. 1966. Antibiotic susceptibility testing standardized single disk method. Am J Clin Pathol 45: 493-496.

5. Chalkley LJ, Koornhof HJ. 1985. Antimicrobial activity of ciprofloxacin against Pseudomonas aeruginosa, Escherichia coli, and Staphylococcus aureus determined by the killing curve method: antibiotic comparisons and synergistic interactions. Antimicrob Agents Chemother 28: 331-342.

6. Cohen ML. 2000. Changing patterns of infectious diseases. Nature 406: 762767. doi: $10.1038 / 35021206$

7. De Francesco K, Cobbold R, Rice D, Besser T, Hancock D. 2004. Antimicrobial resistance of commensal Escherichia coli from dairy cattle associated with recent multi-resistant salmonellosis outbreaks. Vet Microbiol 98: 55-61. doi: 10.1016/j.vetmic.2003.10.017

8. Dobrint U, Agerer F, Michaelis K, Janka A, Buchrieser C, Samuelson M, Svanborg C, et al. 2003. Analysis of genome plasticity in pathogenic and commensal Escherichia coli isolates by use of DNA arrays. J Bacteriol 185: 1831-1840. doi: 10.1128/JB.185.6.18311840.2003

9. Fadda G, Nicoletti G, Schito GC, Tempera G. 2005. Antimicrobial susceptibility patterns of contemporary pathogens from uncomplicated urinary tract infection isolated in a multicenter Italian survey: possible impact in guidelines. J Chemother 17: 251-257. doi: 10.1179/joc.2005.17.3.251

10. Gales AC, Sader HS, Jones RN, SENTRY participants group (Latin America). 2002. Urinary tract infection trends in Latin American hospitals: report from the SENTRY antimicrobial surveillance program (1997-2000).
Diagn Microbiol Infect Dis 44: 289-299. doi: 10.1016/S0732-8893(02)00470-4

11. Gupta N, Sharda R, Sharma V, Deshpande A, Udaykar A. 2011. Pathogenicity and antibiogram of Escherichia coli isolated from diarrhoeic cow calves. Indian J Field Vet 7(2): 1-4.

12. Hendriksen RS, Mevius DJ, Schroeter A, Teale C, Jouy E, Butaye P, Franco, A, et al. 2008. Ocurrence of antimicrobial resistance among bacterial pathogens and indicator bacteria in pigs in different European countries from year 2002-2004: the ARBAO-II study, Acta Vet Scand 50: 19. doi: 10.1186/ 1751-0147-50-19

13. [INS] Instituto Nacional de Salud. 2005. Manual de procedimientos bacteriológicos en infecciones intrahospitalarias. Serie de Normas Técnicas N. ${ }^{\circ} 28$. Lima: INS. 109 p.

14. Iroha IR, Okonkwo EC, Nwakaeze EA, Oji EA, Afiukwa FN, Nwuzo AC, Ayogu TE. 2012. Antimicrobial resistance of Escherichia coli isolated from animal and human clinical sample. Global Res J Microbiol 2(1): 85-89.

15. Lanz R, Kuhnert P, Boerlin P. 2003. Antimicrobial resistance and resistance gene determinants in clinical Escherichia coli isolated from different animal species in Switzerland. Vet Microbiol 91: 73-84. doi: 10.1016/S0378-1135(02) 00263-8

16. Luna L, Maturrano L, Rivera $H$, Zanabria V, Rosadio R. 2012. Genotipificación, evaluación toxigénica in vitro y sensibilidad a antibióticos de cepas de Escherichia coli aisladas de casos diarreicos y fatales en alpacas neonatas. Rev Inv Vet Perú 23: 280-288. doi: 10.15381/rivep.v23i3.910

17. Morales S, Paredes D, Pezo D. 2007. Asociación de rotavirus y Escherichia coli fimbriada como agentes causales de infecciones entéricas en alpacas neonatas. Rev Inv Vet Perú 18: 150-153. doi: 10.15381/rivep.v18i2.1486 
18. Mosquito S, Ruiz J, Bauer J, Ochoa T. 2011. Mecanismos moleculares de resistencia antibiótica en Escherichia coli asociada a diarrea. Rev Peru Med Exp Salud Pública 28: 648-656.

19. [NCCLS] National Committee for Clinical Laboratory Standards. 2003. Performance standards for antimicrobial susceptibility testing: eleventh informational supplement. Wayne, PA, USA: NCCLS Document M100-S11.

20. Sayah RS, Kaneene JB, Johnson Y, Miller RA. 2005. Patterns of antimicrobial resistance observed in Escherichia coli isolates obtained from domestic- and wild-animal fecal samples, human septage, and surface water. Appl Environ Microbiol 71: 1394-1404.
21. Teale CJ, Martin PK, Watkins G. 2002. VLA antimicrobial sensitivity report. UK: Crown.74 p.

22. Thielman NM, Guerrant RL. 1999. Escherichia coli. In: Yu VL, Merigan Jr TC, Barriere SL (eds). Antimicrobial therapy and vaccines. Baltimore, USA: Williams y Wilkins. p 188-200.

23. [WHO] World Health Organization. 2002. The medical impact of the use of antimicrobials in food animals. Report of a WHO meeting publication $\mathrm{WHO} /$ EMC/200/1014. [Internet]. Available in: http://apps.who.int/iris/bitstream/10665/ 64439/1/WHO_EMC_ZOO_97.4.pdf

24. Zahraei T, Farashi S. 2006. Antibiotics susceptibility pattern of Escherichia coli strains isolated from chickens with colisepticemia in Tabriz province, Iran. Internat J Poultry Sci 5: 677-684. 